\title{
Carnets
}

Revue électronique d'études françaises de l'APEF

Deuxième série - 13 | 2018

Corps, rythmes et voix : en/jeux littéraires et artistiques

\section{Du corps de l'acteur a la parole résurrectionnelle}

Approche des écritures agissantes du théâtre de Valère Novarina

\section{Alexandra Gaudechaux}

\section{(2) OpenEdition}

\section{Journals}

Édition électronique

URL : http://journals.openedition.org/carnets/2619

DOI : $10.4000 /$ carnets.2619

ISSN : 1646-7698

Éditeur

APEF

Référence électronique

Alexandra Gaudechaux, «Du corps de l'acteur a la parole résurrectionnelle», Carnets [En ligne], Deuxième série - 13 | 2018, mis en ligne le 31 mai 2018, consulté le 01 mai 2019. URL : http:// journals.openedition.org/carnets/2619; DOI : 10.4000/carnets.2619

Ce document a été généré automatiquement le 1 mai 2019.

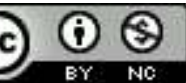

Carnets est mis à disposition selon les termes de la licence Creative Commons - Atribution - Pas d'utilisation commerciale 4.0 International. 


\title{
Du corps de l'acteur a la parole résurrectionnelle
}

\author{
Approche des écritures agissantes du théâtre de Valère Novarina
}

\author{
Alexandra Gaudechaux
}

1 Les écritures dramatiques de Valère Novarina ont de quoi décontenancer jusqu'aux lecteurs les plus aguerris. C'est tout d'abord l'extension d'un texte qui semble se combiner à l'infini qui interpelle, fruit d'un travail du souffle mais aussi de l'écoute attentive au moment de l'écriture, poursuivant ce que l'auteur désigne en tant que « voix qui murmure» (O'Byrne, 2002), dialogue avec la création, si ce n'est appel au surgissement d'une parole jusqu'alors silencieuse. Il ne s'agit plus pour le lecteur dès lors de s'en remettre à une quelconque pérégrination intimiste ni de s'épancher sur les troubles de la psyché et autres vagues à l'âme personnels mais bien au contraire de se distancer des fantasmes de l'identification au profit d'une écoute dont l'auteur se préfère l'humble serviteur, le médiateur scrupuleux. Voici où la voix se meut, en amont déjà de toute interprétation par l'acteur et au commencement même du geste d'écriture. D'où également un acte d'écrire selon Valère Novarina qui se doit de favoriser " l'autre voix » de l'écriture (Steinmetz, 1990 : 95), celle par exemple dont Philippe Jaccottet nous confie la prégnance dans La Seconde Semaison ${ }^{1}$ à propos de Céline et de son Voyage: dialogue généreux entre voix poétique et voix musicale où l'écoute attentive de l'écrivain « porte le deuil d'autres voix qui affleurent et le traversent de leur signifiance inouïe et muette » (Jaccottet, $1985: 29$ ). Relevons pour souligner ce parallèle entre voix poétique et parole chantée l'un des passages extrait d'Entrée perpétuelle, une version pour la scène du Vivier des noms où l'auteur nous invite à ce passage subtil - vécu néanmoins comme une évidence - du langage parlé au chanté :

AUCUN

Voici les cendres de mon père, les cendres de ma mère: je les mélange, et je n'apparais point. Je les secoue : me voici moi. Je les divise : voici les poussières de mon fils arrière, et les cendres de l'arrière-petit-fils de mon prochain grand-père. Je les mélange en une immense boîte à joies et je les verse une à une à la poubelle dans la nuit. Choses parmi-là qui sont ici, disparpillez-vous en un sac ; puis réunificatezvous en mille semailles ! Ce qui est semé en mépris, ressuscitera en gloire ! 
Chanté:

Les chiffres disparaîtront. Les chiffres sont l'excrément du temps. Quand tu comptes, tu énumères à l'envers le nombre des chiffres passés par là ! Quand tu comptes, tu remontes du temps, tu croises des chiffres en traversant! Les chiffres nous protègent de l'effrayante vie : merci les chiffres! Deux et deux sont quatre! Soixante-deux fois trois font cent-quatre-vingt-six! Sept cent quatre-vingt-huit mille sept cent cinquante-trois deux fois sont un million cinq cent soixante-dix-sept mille cinq cent six ! Ah oui, merci les chiffres huit ${ }^{2}$ !...

2 De ce premier passage, notons tout d'abord sa référence aux psaumes chrétiens qu'il semble ici fondamental de préciser tant sa teneur se veut l'une des constituantes principales à la " génétique » des écritures novariniennes. Si le psaume se désigne comme texte poétique composé de plusieurs versets, il relève aussi de la liturgie de la Parole, prière quotidienne des chrétiens composée des textes de la sainte Ecriture et pendant laquelle vont s'intercaler des chants favorisant la méditation. Ce rituel religieux destiné à l'accueil de la parole divine bénéficie sous la plume novarinienne de sémantiques plurielles qui invitent l'auditeur à ce voyage hors de soi qu'Emmanuel Lévinas appelait "évasion " ${ }^{3}$. Ainsi précisé par le philosophe de Totalité et infini ${ }^{4}$, il ne s'agit plus pour l'individu de s'absenter quelques instants de lui-même par un subterfuge prévisible mais bien plutôt d'atteindre cette "évasion sans itinéraire et sans termes " qui ne vise dès lors plus à "accoster quelque part» mais "partir pour partir» (Lévinas, 1977: 151). Il existe néanmoins un retour inévitable, bien sûr. Cependant ce retour est comme neuf, loin de la signifiance qui communique et où désormais peuvent consteller les équivoques de sens, la fraîcheur du poème comme lieu de renaissances. Cet appel à l'« autre en soi » mais aussi à l'« énigme de l'altérité » (Guénoun, 2015: 50), Valère Novarina le fait incarner par «Aucun » et le mythe fondateur de l'engendrement christique qu'il suggère.

Notons en effet que le psaume se décline sous la forme d'une "Pastorale " relative à la nativité par laquelle l'individu « appelé » par le Christ - pour reprendre ici la définition proposée par Gabriel Monet ${ }^{5}$ - accède à sa renaissance par le biais du Verbe et de son incarnation. La parole animée du souffle de l'acteur y trouve dès lors une place centrale, seule capable d'engendrer cet Homme hors de lui ${ }^{6}$ auquel Valère Novarina confie le titre de sa nouvelle création pour la scène, interprétée pour la première fois le 20 septembre 2017, au Théatre de la Colline par le comédien Dominique Pinon. Ces indices confirment à l'endroit des écrits de Valère Novarina ce qu'Olivier Dubouclez nomme aussi "l'agissement d'une poétique théologique » (Dubouclez, 2014:1) portant à réconcilier l'humain avec ce qu'il est de sacré : «révélation de l'homme par l'homme » (Mondzain, 2017: préface). Une théologie dont l'auteur nous dévoile en outre les aspirations libératrices dans la mesure où ces «Pères de l'Eglise, mystiques et théologiens cherch [ai]ent par tous les moyens de la parole et de la raison (...) une vérité qui ne se capture pas» (Dubouclez, 2014: 10). La rencontre de Valère Novarina avec la littérature spirituelle fut initiée par son voyage fasciné dans ce qu'il nomme «la nuit noire du Moyen-Âge » (ibid : 11), recelant un trésor jusqu'alors tenu pour secret. S'en suit une passion quasiment obsessionnelle du jeune étudiant qu'il était alors pour les théologiens du haut Moyen-Âge, lesquels, plutôt que d'enfermer le langage dans une équivalence plane entre signifiant et signifié, proposaient déjà de multiples innovations linguistiques destinées à transfigurer la langue. Traversé physiquement et spirituellement par cette aventure philologique, Valère Novarina commentera sa relation aux écrits des théologiens comme s'il était parcouru de fièvre alors que se succédaient entre ses mains 
les textes d'Athanase d'Alexandrie, Rupert de Deutz, Méliton de Sardes, Marius Victorinus ou encore Hilaire de Poitiers, dont les noms aux consonances lointaines attiraient l'auteur avant même d'en découvrir la prose. Nous retrouvons parmi ces lectures le goût de Novarina pour ce qu'il nomme les "passages nouveaux » de l'écriture, capables de transporter son lecteur dans des voyages " jusqu'au bout de la langue. ${ }^{7}$ ", Au-delà du style foisonnant des auteurs du Moyen-Âge, Novarina y découvre l'expression d'une libre pensée capable de défier les lois de la logique et les limites de la raison.

Ceux qu'il qualifie de théologiens philosophes incarnent alors à ses yeux un modèle inédit dont la pensée subversive parvient à déjouer le langage et ses prétentions. Ces « poètes de la pensée » (De Courcelles, 2006: 5), dont Valère Novarina peut en toute légitimité revendiquer l'héritage, incarnent dès lors une audace à la mesure de son génie, dont la quête désormais commune se destine au dévoilement des choses tues: définir l'impalpable, cerner l'impensable ou encore "résoudre de surnaturelles équations mathématiques » (ibid). Nous retrouvons dans les écrits de Valère Novarina cette quête de l'impossible à l'endroit du langage, accordant à sa pensée une force poétique et spirituelle inédites. C'est aussi pourquoi il serait vain et sans intérêt de vouloir séparer le poème de ses résonances théologiques, tant l'un et l'autre se répondent et donnent lieu à ce qu'Olivier Dubouclez nomme avec pertinence «une pensée du divin travaillée par Novarina sur le mode du poème» (Dubouclez, 2014: 12). Un transport qui, en outre, favorise le vocable sacré, par essence imprononçable et inaudible. Parmi ces quêtes de l'impossible, nous retrouvons Valère Novarina en aventurier de la pensée ou encore "scientifique sans espoir» (ibid) - tel qu'il aime à se désigner lui-même - parvenant à théoriser une pensée génialement digressive dont les schémas réalisés dans le but d' «éclaircir l'obscure page cinquante-huit de l'Envers de l'esprit » (Novarina, 2012: 133) évoquent fortement les intuitions tout aussi géniales d'un alchimiste de l'époque médiévale. Cependant, si l'alchimiste se donnait pour mission de transformer le plomb en or, le projet de Valère Novarina se destine bien plutôt à « creuser » (Novarina, 2010 : 65) les mystères de la parole en tant que "physique des fluides capable d'anamorphoses" (ibid). C'est ainsi à la page 134 de La Quatrième Personne du singulier ${ }^{8}$ que nous découvrons le schéma explicatif de cette intuition, définie également en tant que théorie des perspectives croisées, laquelle tente de mettre au clair ce que Novarina nomme le «transvasement de la communication indirecte » (ibid). En véritable maitre d'oeuvre, l'auteur nous informe en outre d'une logique implacable et « rythmiquement juste » (ibid : 135) dont ses écrits pour la scène se portent garants, capables d'opérer sur le spectateur cette action de voyance inédite rendant perceptible à tous - mais selon des voies intimes et singulières - ce que Valère Novarina appelle : l'incarnation du Verbe. Un phénomène qui cependant n'entre dans aucun systématisme et dès lors ne s'observe, selon les dires de l'auteur, qu'en de rares instants où « tout sonne vrai » (ibid : 15).

Outre des références théologiques de l'extrait précédemment cité, nous y observons une savoureuse ambivalence comique oeuvrant à ce qu'olivier Dubouclez nomme la «fusion du comique et de la sainteté »(Dubouclez, 2014: 2). Cette athéologie typiquement novarinienne où néologismes, hyperboles et autres accumulations numériques contribuent au caractère risible du personnage, inaugure surtout une "théologie négative» (ibid: 5) où se jouent ce que Valère Novarina nomme le Niement. Une théorie capable de traverser la matière et à travers elle, peut-être, de répondre à la question du dire et de ses limites. Revenons sur ces termes qui nous permettront de creuser encore davantage la matière vive novarinienne en tant que « poésie » aspirant au passage à l'acte 
(Novarina, 1991: 76). Le concept de niement - développé par Valère Novarina dans son dernier recueil théorique intitulé Voie négative ${ }^{9}$ - s'inscrit dans le sillage de la théologie négative issue de l'époque médiévale. Si l'on reprend sa définition classique tout d'abord, ce concept s'inspire de l'apophatisme : issu du grec aphairesis signifiant «abstraction », il consiste en une méthode de pensée qui se propose de concevoir Dieu en annulant toute possibilité de discours à son égard. La théologie se déclinant en effet comme discours sur le divin, une démarche apophatique se comprend dès lors comme ambition de l'esprit à atteindre la transcendance par l'entremise de propositions négatives. Une dynamique de la transgression d'une part, mais aussi de l'inversion des valeurs d'autre part, qui chez Valère Novarina se traduit en une mise en pièces scrupuleuse de la condition propre au langage humain en ce qu'il se révèle incapable d'exprimer par le langage ce qui s'exprime dans le langage. En cela, l'apophatisme, ou encore niement, se déclinent avant tout comme « signe de l'indicible mystère de l'existence » (Boblet, $2006: 8$ ). C'est aussi pourquoi nous retrouvons les grands théologiens du haut Moyen-Âge tels qu'abordés plus haut, dont l'ambition fut de dépasser les limites du dire dans le but de nommer Dieu. L'écriture de Valère Novarina illustre dès lors - et par cet extrait notamment - le recours à un répertoire théologique européen regorgeant de formules audacieuses, inattendues ou incompréhensibles, capables de hisser le langage jusqu'en cette dimension supérieure et transcendantale, seule capable de rompre avec toute possibilité de communication. Ce qu'Olvier Dubouclez et d'autres grands penseurs de l'oeuvre novarinienne désigneront aussi en tant que "théopoésie » (ibid: 1 ). La pensée, dès lors, perd tous ses repères et tente de se frayer un passage laborieux entre métaphores et hyperboles.

6 S'y actualise également le mythe de la Genèse ou cette foisonnante Seconde Semaison ${ }^{10}$ proposée par Philippe Jaccottet, telle une renaissance appelée et perpétuelle où le nonsavoir des choses vues et ressenties s'y préfère à leur connaissance encyclopédique. Une ode au mystère de cet "autre monde» (Steinmetz, 2006: 15) qui plonge l'homme dans l'immédiateté de son émotion. Lorsque la parole se dérobe à cet état d'urgence survient ce que Jean-Luc Steinmetz nomme pertinemment une «irruption du sacré »(ibid) à l'intérieur des mots, phénoménalité exceptionnelle dont seule la poésie semble porteuse. Entre floraison et reprise incessante de paysages rêvés ou lus, l'écriture arborescente de Valère Novarina nous invite ainsi à franchir nos "étroites limites" (ibid: 21) et nous ouvre des perceptions inédites dans les intervalles du langage, telles ces «fleurs du laurier-rose » inspirant à Philippe Jaccottet la prose poétique qui suit :

Les fleurs du laurier-rose toujours fleuries, depuis des semaines - si mystérieuses pour peu qu'on y pense. Pourquoi a-t-il fallu qu'il y ait des fleurs - des couleurs? Leur rose - sans pareil : une fraîcheur. Ou comme quand les enfants portent des lanternes éclairées, pour des fêtes. Lanternes en plein jour. Mais aussi efflorescences de la terre, métamorphose, la monnaie, la petite monnaie des graines 11 .

7 Tout comme de l'arbre patientant l'hiver pour fleurir et s'épanouir, puisant de la terre les forces nécessaires à son ascension ainsi que des rayons du soleil, les écritures de Valère Novarina procèdent d'une dynamique organique : des racines du langage aux cimes des signifiances, oeuvrant par allers et retours tel l'artisan à son ouvrage en quête de visions inédites. C'est ainsi que l'auteur nous confie procéder par « creusements dans le 'puits du langage'" (Novarina, 2010:13) afin de dire toujours autrement notre rapport au monde, nier les évidences et ses slogans si ce n'est retrouver « ce noyau dur de l'humain qu'est la parole » (Maulpoix, 2010: 3). Ainsi du poète aussi, qui selon Jean-Michel Maulpoix «traverse l'ignorance et accuse l'impensable du réel» (Maulpoix, 2001: 5). Valère 
Novarina choisit de travailler inlassablement la partition textuelle, s'attèle à sa table d'écrivain et sonde la matière du langage pour qu'en adviennent d'infinies perspectives.

Un auteur, enfin, dont nous pouvons observer l'artisanat " perplexe » (ibid) - de perplexus en latin signifiant "enlacé, enchevêtré, confondu» mais aussi de façon figurée « embarrassé et obscur » - à l'endroit d'un langage poétique comme musique capable de «creuser l'espace pour [faire] comprendre une nouvelle langue» (Thomasseau, 2002: 165). Les écrits de Valère Novarina se fondent à partir de ce que Céline Hersant nomme un " geste d'écriture » (Hersant, 2015 : 10), geste dirigé depuis l'espace de la page jusqu'à celui des murs de son atelier, étendu à celui de l'espace mental du spectateur. Ainsi, la parole novarinienne se décline comme lieu d'échange et offrande capables de toucher l'auditeur dans ce qu'il a de plus intime : son langage. La parole se veut ainsi « approche délicate de l'autre » (Derrida, $2000: 15$ ), déplacement hors de soi comme en état de veille que nous évoque cette " phase nocturne d'écriture » (Perrier, $2007: 1$ ) à laquelle s'adonne l'auteur Novarina en amont du livre. Ce retrait de soi dans l'attente et l'écoute attentive de ce qui n'est pas encore, nous le retrouvons aussi dans la philosophie de la caresse selon Emmanuel Lévinas. Une caresse qui est aussi attente qui consiste pourtant à « ne se saisir de rien, solliciter ce qui s'échappe sans cesse vers un avenir. La caresse cherche ce qui n'est pas encore» (Lévinas, $1980: 235$ ). Une attitude que nous pouvons rapprocher de celle opérée par Valère Novarina au moment de l'écriture, lorsque de la pensée qui veille peut advenir une parole spontanée, tel un élan vital dépourvu de signification logique mais d'autant plus réel qu'il se veut générosité et dessaisissement de soi. Une attitude qui fait alors écho à la kénose de l'incarnation, laquelle consiste en l'anéantissement de l'individu pendant le phénomène d'incarnation du Verbe, et ce dans le but de dépasser sa propre condition mortelle vers celle, divine, du Christ. Une mise en veille de soi dans l'attente d'un langage "surgissant» (Novarina, 2012: 120) qui mènerait surtout à provoquer chez celui qu'il traverse ce que Novarina nomme un "sur'lèvement » (ibid) ou encore anagogie, cette notion ascétique désignant l'élévation de l'âme vers les choses célestes. Une réflexion que l'on retrouve dans l'essai théorique que Valère Novarin rédigea en 1999 comme suite à ses 616 notes de Pendant la Matière :

Penser, c'est attendre en pensée, avoir corps et esprit en accueil. La pensée de saisit pas, ne possède rien; elle veille, elle attend. De même parler, ce n'est pas avoir quelque chose à dire et savoir s'exprimer, mais c'est attendre aussi la parole ${ }^{12}$.

L'esthétique de l'écriture novarinienne se rapprocherait dès lors de ce qu'Amodor Vega nomme la « mystique médiévale » (De Courcelles, 2007), notamment en ce qu'elle désigne un auteur qui refuse de prétendre à une position d'autorité et dont nous retrouvons l'humilité à travers celle de Michel de Certeau dans son introduction à La Fable mystique. Observons l'extrait ci-après afin d'y apprécier ce glissement entre les deux auteurs : « Ce livre se présente au nom d'une incompétence : il est exilé de ce qu'il traite. L'écriture que je dédie aux discours mystiques de (ou sur) la présence (de Dieu) a pour statut de ne pas être » (De Certeau, 1987 : 9). Une double distance pudique entre celui qui écrit et son objet d'écriture qui dévoile en outre une quête à dire l'autre que seul «l'exercice d'absence à soi-même» (ibid : 309) - propre aux mystiques de l'antiquité - semble favoriser. Exercice que Valère Novarina nomme à son tour «cure d'idiotie » (Novarina, 1986:5) et que nous comprenons dès lors à la fois comme l'opération par laquelle l'auteur peut produire son texte, mais aussi seule pratique capable de garantir le surgissement d'une pensée déliante ou ce que Dominique de Courcelles nomme une "poésie de la pensée " (De Courcelles: ibid). Parmi ces grands praticiens de la mystique médiévale figurent notamment Raymond Lulle, Bonaventure ou encore Maître Eckhart, auteurs dont Valère Novarina étudia les 
ouvrages et dont nous constatons encore l'influence à l'occasion de cet autre extrait de La Quatrième Personne du Singulier, où pensée et poésie se côtoient avec virtuosité :

(...) nous sommes dénoués par le Verbe, sortis des liens de la mort, des bandelettes enchaînantes de nos idées d'automates, de nos rêveries tournantes, de nos cercles mentaux. La pensée est un organisme. La pensée est un animal délivrant ${ }^{13}$.

Musique de la syntaxe et des rythmes de la ponctuation, jeux d'équivoques par les mots et leurs polysémies virevoltantes afin de provoquer le déséquilibre de l'entendement, l'écriture novarinienne se veut aussi « délivrante » et "porteuse de paroles » (Novarina, 1999: 16), à commencer par celles issues de langues classées «mortes", dialectes régionaux et autres paroles souterraines. Son écriture - que l'auteur préfère en cela conjuguer au pluriel - s'apparente ainsi davantage à une forme de résistance au service des multiples formes de l'oralité - d'où sa portée théâtrale - appelant à ce qu'elles renvoient à l'espace de la page, puis de la respiration chez l'acteur. Une écriture qui n'est ainsi en rien l'expression figée de sophistications linguistiques ni la répétition de procédés mercantiles.

11 L'esthétique de l'écriture novarinienne s'éloigne en cela véritablement de l'héritage traditionnel du théâtre bourgeois, y préférant la réinvention permanente de ses propres codes. L'écriture se transforme alors en musique, langage différent par lequel il faut se laisser porter afin d'y déposer l'animal raisonnant que nous sommes. Le logos, pourtant maître de notre pensée, se dissipe peu à peu et laisse place à l'autre voix en nous : et à celle de la poésie. Une écriture, ainsi, qui de par ses modalités d'entendement inédites fait résonner des façons de parler méconnues et hybrides, issues des «manières " propres à ceux que l'auteur croisa sur son chemin et dont il enregistra mentalement les expressions, selon un «style » propre à chaque individu parlant qu'il précise comme suit, à l'occasion d'une rencontre modérée par Marion Ferry à l'Odéon-Théâtre de l'Europe en 2011 : « Chacun a sa nage. Son rapport rythmique profond avec sa langue (...) Il me semble que chacun a son propre langage, son style, ses mots favoris. " D'où également l'affirmation d'une langue qui se doit d'être singulière, par essence, tant elle se veut selon Valère Novarina l'expression d'une individualité propre à chacun, la singularité d'une respiration. Écrire se décline dès lors comme voix et rythmes intérieurs de l'écrivain; ce que l'on retrouve aussi chez les auteurs qualifiés de «difficiles». Valère Novarina cite pour exemple sa lecture des œuvres de Bossuet ou de Céline, qui impliquèrent à l'époque une véritable épreuve du rythme pour le lecteur précoce qu'il était. C'est ainsi par la compréhension intime, si ce n'est intuitive, de la respiration de ces écrivains qu'il lui fut possible de lire leurs œuvres magistrales, suivant ce lien tacite que l'auteur crée de son souffle à travers ce que Jean-Luc Steinmetz nomme "le médium léger de l'écriture » (Steinmetz, 1990 : 13). Et de poursuivre à cet égard en précisant que c'est «tout à coup » qu'il comprit « comment Bossuet respirait » (Ferry, 2011). Un rapport qui se veut dès lors au plus proche de l'écrivain, cherchant bien plus que de réduire le sens de ses écrits en une compréhension totalisante - et donc incomplète - , à opérer en soi un déplacement intellectuel mais surtout affectif, capable d'entrer en rapport avec cet «autre» de l'écriture, ou ce que nous nommions encore la sympathie (Bergson, $1907: 10)$ de l'élan intuitif. Pour Novarina, l'individu se fait dès lors inventeur de sa propre langue, tels les nombreux dialectes patois qui parcoururent les paysages de son enfance savoyarde et gravèrent dans l'esprit du jeune écrivain la conviction qu'il existe autant de langues qu'il est d'hommes, et que la traversée du langage se veut constitutive de tout individu dans ce qu'il est de mémoires et d'héritages. Une résistance qui permet ainsi de s'inventer soi- 
même dans l'action de parler, et d'y ouvrir, par ce biais, un contact essentiel avec sa propre différence.

\section{BIBLIOGRAPHIE}

BERGSON, Henri (1907). L'évolution créatrice. Paris : Félix Alcan.

BOBLET, Marie-Hélène (2006). « Le théâtre éphâtique de Valère Novarina, Le tact de la parole », Revue d'Histoire Littéraire de la France, vol. 106.

CASTEIG, Julie (2006). Connaissance et vérité chez Maître Eckhart. Seul le juste connaît la justice, coll. «Etudes de philosophie médiévale ». Paris : Vrin.

CELINE, Louis-Ferdinand (1932). Voyage au bout de la nuit. Paris : Denoël et Steele.

DERRIDA, Jacques (2000). Le Toucher, Jean-Luc Nancy. Paris : Galilée.

PHILIPPON, Michel (1999). «Valéry et la poétique de l'indéterminé : lire le poème -lyre », in Valérie-Angélique Deshoulières (dir.) Poétiques de l'indéterminé. Le caméléon au propre comme au figuré. Clermont-Ferrand : Presses de l'Université Blaise Pascal (Littératures).

DE CERTEAU, Michel (1987). La Fable mystique, 1 : XVI $I^{e}-X V I I^{e}$ siècle. Paris : Poche.

DE COURCELLES, Dominique (1993). La parole risquée de Raymond Lulle : entre judaïsme, christianisme et islam. Paris : Vrin.

DE COURCELlES, Dominique (dir.) (2007). Les enjeux philosophiques de la mystique, Actes du colloque du Collège international de philosophie, 6-8 avril 2006, Grenoble : Éditions Jérôme Millon.

DUBOUCLEZ, Olivier et James, Alison (2014). « Valère Novarina : une poétique théologique.

Introduction », Revue de Littérature, 176.

GILSON, Etienne (1953). La philosophie de saint Bonaventure. Paris : Vrin.

GUENOUN, Tamara (2015). « Le personnage, figure de l'autre en soi », L'Esprit du temps, Recherches en psychanalyse, $\mathrm{n}^{\circ} 19$.

HERSANT, Céline (2015). L'atelier de Valère Novarina : recyclage et fabrique continue du texte. Paris : Garnier.

JAccottet, Philippe (1996). La Seconde Semaison. Carnets (1980-1994), Collection Blanche. Paris : Gallimard.

LEVINAS, Emmanuel (1980), Totalité et Infini. Essai sur l'extériorité. La Haye, Boston, Londres :

Martinus Nijhoff Publishers.

LEVINAS, Emmanuel (1982). De l'évasion. Saint Clément de rivière : Fata Morgana.

LEVINAS, Emmanuel (1947/1977). De l'existence à l'existant. Paris : Vrin.

LE BRUN, Jacques (2004). « La mystique et ses histoires », in Revue de théologie et de philosophie, 136, p. 309-318. 
NOVARINA, Valère (2002). Ce dont on ne peut parler c'est cela qu'il faut dire, film documentaire réalisé par Raphaël O'Byrne, coproduction Arte France, Les films à Lou.

MAULPoIX, Jean-Michel (2001). Le poète perplexe. Essai critique. Paris : Éditions José Corti.

MAULPOIX, Jean-Michel (2010). « La parole suractive. Essai sur Devant la parole (P.O.L), de Valère Novarina ». Paris : Éditions José Corti.

MONDZAIN, Marie-José (2017). Préface au spectacle « L'Homme hors de lui », op.cit.

MONET, Gabriel (2010). La Pastorale d'engendrement : appel à un regard chrétien nouveau sur la naissance et le développement de la foi, Études Chrétiennes.

NOVARINA, Valère (1999). Devant la parole. Paris : P.O.L.

NOVARINA, Valère (2012). La Quatrième Personne du Singulier. Paris : P.O.L.

NOVARINA, Valère (1991). Pendant la Matière. Paris : P.O.L.

NOVARINA, Valère (2010). Lumières du corps. Paris : P.O.L.

NOVARINA, Valère (2017). Voie négative. Paris : P.O.L.

PERRIER, Jean-François (2007). Entretien avec Valère Novarina.

STEINMETz. Jean-Luc (1990). «Entre proche et lointain : 1' 'autre chose' de Philippe Jaccottet », in Michel Collot et Jean-Claude Mathieu (dir.) Poésie et Altérité. Paris : Éd. Rue d'Ulm.

THOMASSEAU, Jean-Marie (2002). « Préface à « L'homme hors de lui » de Valère Novarina », Europe, $\mathrm{n}^{\circ} 880-881$.

VASSEUR, Fabien (2002). « Jaccottet, voix de Purcell », Percée Littérature, Volume 127, Numéro 3, pp. 19-28.

\section{NOTES}

1. JACCOTTET Philippe, La Seconde Semaison, Carnets (1980-1994), Gallimard, 1985, p. 29.

2. NOVARINA Valère, Voie négative, «Entrée perpétuelle ", version pour la scène du Vivier des noms, P.O.L, 2017, p. 146.

3. LEVINAS Emmanuel, De l'évasion, Fata Morgana, 1982.

4. LEVINAS Emmanuel, Totalité et infini. Essai sur l'extériorité, Martinus Nijhoff, 1961.

5. MONET Gabriel, La Pastorale d'engendrement : appel à un regard chrétien nouveau sur la naissance et le développement de la foi, Études Chrétiennes, Mai 2010.

6. NOVARINA Valère, L'Homme hors de lui, écrit et mis en scène par Valère Novarina et interprété par Dominique Pinon, La Colline Théâtre National, du 20 septembre au 19 octobre 2017.

7. QUIGNARD Pascal, Le nom sur le bout de la langue, P.O.L, 1993.

8. NOVARINA Valère, La Quatrième Personne du Singulier, P.O.L, 2012.

9. NOVARINA Valère, Voie négative, P.O.L, 2017.

10. JACCOTTET Philippe, La Seconde Semaison. Carnets (1980-1994), Coll. Blanche, Gallimard, 1996.

11. op.cit., p. 10.

12. NOVARINA Valère, Pendant la Matière, P.O.L, 1991, p. 26.

13. NOVARINA Valère, La Quatrième Personne du Singulier, P.O.L, 2012, p. 126. 


\section{RÉSUMÉS}

L'oeuvre théâtrale de Valère Novarina est une langue qui vous renverse. Pluriel et jubilatoire, son art de l'écriture opère en véritable artisanat de l'inouï porté par « l'organe du langage [qu']est la main» (Ferry, 2011). Un équilibre favorisant l'efficience d'une poétique de l'indéterminé et qui renoue en cela avec la phénoménale fécondité du Verbe telle qu'initiée par les théologiens du haut Moyen-Âge. Une parole que l'on peut aussi rapprocher du valéry - cette langue si difficile dont le poète lui-même en ignore l'apprentissage - et de l'écriture hébraïque, lesquelles ont en commun d'offrir à son lecteur des «vides à remplir, [des] lacunes à combler pour le son comme pour le sens » (Philippon, 1999 : 349), le tout oeuvrant à la transfiguration des carences du langage en richesses du poème (ibid). La parole novarinienne jaillit et ravive tel un fluide agissant le corps des acteurs en des reconfigurations infinies de la figure humaine. Nous nous proposons ici d'en observer quelques passages.

The theatrical work of Valere Novarina is a language that turns you upside down. Plural and jubilant, his art of writing operates as a real craft of the unheard of "the organ of language [that] is the hand." An equilibrium favoring the efficiency of a poetics of the indeterminate, which is related to the phenomenal fruitfulness of the Word as initiated by the theologians of the Middle Ages. A word that can also be related to valery - the language so "difficult" that the poet himself ignores learning - and Hebrew writing, which have in common to offer his reader "empty" to fill, [gaps] to fill for the sound as for the sense ", the whole working to the transfiguration of" deficiencies "of the language in" riches of the poem ". The Novarin language springs up and revives like a fluid acting the actors' bodies in infinite reconfigurations of the human figure. We propose here to observe some passages.

\section{INDEX}

Mots-clés : Novarina (Valère), écriture dramatique, corps de l'acteur, réception théâtrale, théologie médiévale

Keywords : Novarina (Valère), dramatic writing, actor's body, theatrical reception, medieval theology

\section{AUTEUR}

\section{ALEXANDRA GAUDECHAUX}

Université de Haute Bretagne, Rennes 2

alexandra.gaudechaux[at]@univ-rennes2.fr 